\section{Avaliação normativa das ações do Programa de Atenção Integral à Saúde da Criança (PAISC) em Pernambuco}

\section{Normative evaluation of the Whole Attention to Child Health Program (WACHP) in Pernambuco}

Suely Arruda Vidal 1

Paulo Germano de Frias 2

Neusa Maria Marques 3

1 Instituto Materno Infantil de Pernambuco, IMIP Rua dos Coelhos, 300, Boa Vista, Recife, Pernambuco, Brasil. CEP 50.070-550

e-mail: suelyarruda@hotmail.com

2 Mestrado da Universidade Federal de Pernambuco

3 Departamento Materno Infantil da Universidade

Federal de Pernambuco

\begin{abstract}
Objectives: A descriptive study was carried out to evaluate the implementation of the Whole Attention to Child Health Program (WACHP) in the state of Pernambuco, Brazil.

Methods: one health unit in each of the $176 \mathrm{mu}$ nicipalities and 6 units in the capital city of the state were selected for the study. The evaluation was based on the guidelines for evaluation of health programs as recommended by the Ministry of Health. Data collection took place from October to December 1995.

Results: the findings show that, in general, the program was poorly implemented. Program implementation was better for the immunization and breastfeeding components in the State as a whole and for the breastfeeding, growth and development and immunization components in the capital city .

Conclusions: the findings were discussed in the light of the findings of the Second Health and Nutrition Survey for the state of Pernambuco, as well as of other studies carried out in the same field, which suggested that the WACHP implementation should be improved.
\end{abstract}

Key words Assessment of health services and programmes; Child health, Breast feeding, Immunization, Child development

\section{Resumo}

Objetivos: avaliar o grau de implantação do Programa de Atenção Integral à Saúde da Criança (PAISC), em Pernambuco.

Métodos: estudo descritivo-avaliativo, de corte transversal, realizado nas unidades de saúde de maior complexidade dos 176 municípios do estado, exceção feita à capital, onde foram escolhidas seis unidades de saúde e analisadas separadamente. A coleta dos dados se processou no período de outubro a dezembro de 1995, e teve como instrumento o "Roteiro de Avaliação dos Programas" do Ministério da Saúde.

Resultados: observou-se um baixo grau de implantação para o conjunto das ações programáticas do PAISC. A melhor performance, para o estado de Pernambuco, foi verificada nas ações de Imunização e Incentivo ao Aleitamento Materno. Para o Recife, o destaque foi para o Incentivo ao Aleitamento Materno, seguido do Acompanhamento do Crescimento e Desenvolvimento e Imunização, ambos com o mesmo percentual. Estes resultados foram confrontados com os encontrados na II Pesquisa Estadual de Saúde e Nutrição, Pernambuco, e de outros estudos nesta área, e sugerem que as ações básicas de saúde necessitam ainda ser melhor trabalhadas.

Conclusões: os dados foram discutidos à luz da II Pesquisa Estadual de Saúde e Nutrição, Pernambuco, bem como de outros estudos realizados no mesmo campo e sugerem que a aplicação do PAISC deve ser melhorada.

Palavras-chave Avaliação de serviços e programas; Saúde da criança, Aleitamento materno, Imunização, Desenvolvimento infantil 


\section{Introdução}

Em 1986 o Ministério da Saúde do Brasil1 implantou o Programa de Assistência Integral à Saúde da Mulher, Criança e Adolescente (PAISMC/PROSAD) - com o objetivo de reduzir a morbi-mortalidade neste grupo populacional através das ações básicas de saúde. O programa da criança (PAISC) visa melhorar as condições de assistência à saúde, na faixa etária de 0 a 5 anos, com prioridade para grupos de risco. É composto pelas ações de Acompanhamento do Crescimento e Desenvolvimento, Incentivo ao Aleitamento Materno e Orientação para o Desmame, Controle das Doenças Diarréicas, Controle das Infecções Respiratórias Agudas e Imunização.

Através destas ações é possível cumprir um grupo de metas estabelecidas pela Reunião de Cúpula em Favor da Infância, ocorrida em Nova York em 1990, a serem atingidas até o ano 2000. O Brasil foi um dos participantes desta reunião e um dos 158 signatários da Declaração Mundial sobre Sobrevivência, Proteção e Desenvolvimento da Criança. ${ }^{2}$ Para verificação do cumprimento das metas, o Ministério da Saúde do Brasil utilizou pesquisas de base populacional, por permitir a aquisição de informações complementares às dos serviços, e também em virtude da fragilidade dos sistemas, considerando a cobertura, qualidade e pertinência dos dados.

No estado de Pernambuco foi utilizada a I Pesquisa Estadual de Saúde e Nutrição (I PESN) realizada em 1992, que descreve as condições de saúde das mulheres e crianças. Os resultados encontrados foram desfavoráveis em relação à prevalência do aleitamento materno, uso da Terapia de Reidratação Oral (TRO), do "cartão da criança", desnutrição e anemia. ${ }^{3}$

Diante disso foi identificada a necessidade de se realizar uma avaliação normativa que tem as funções de controle e acompanhamento e os resultados são úteis para contribuir com a reorientação das ações ou da intervenção. Este tipo de avaliação " ... se apoia no postulado de que existe uma relação forte entre o respeito aos critérios e às normas escolhidas e os efeitos reais do programa". (Contandriopoulos et al.; 1992; 35). 4

Para tanto, a Secretaria de Saúde do Estado buscou conhecer a situação das ações do Programa de Atenção Integral à Saúde da Criança (PAISC) em cada um dos seus municípios.

\section{Métodos}

Foi realizado um estudo descritivo de corte transver- sal, no período de outubro a dezembro de 1995 . Foi selecionada uma unidade de referência ambulatorial (a de maior complexidade existente) de cada município de Pernambuco (176). Na capital, Recife, optouse por selecionar uma unidade de referência em cada um dos seis distritos sanitários, em virtude da complexidade da rede de serviços de saúde, assim como do número de habitantes, similar a dos maiores municípios do estado.

Esta escolha deveu-se à necessidade da Secretaria Estadual de Saúde de obter uma resposta rápida do panorama do PAISC e a impossibilidade de se avaliar em pouco tempo todas as unidades. Utilizouse como argumento o fato de que as melhores situações deveriam estar nas unidades de referência. Foi empregado um instrumento padronizado pelo Ministério da Saúde,5 em 1994, denominado "Roteiro de Avaliação dos Programas". Este contempla questões relacionadas às normas das ações básicas de atenção à saúde da criança, mulher e adolescente, do qual, neste artigo, foram analisadas apenas as cinco ações básicas do programa da criança.

De acordo com Contandriopoulos et al.6, as vantagens em se utilizar um instrumento já conhecido seriam a possibilidade de comparação, a praticidade do instrumento e a redução do tempo e do custo da avaliação. Também havia a facilidade de consolidação e análise dos dados, através do "Caderno de Respostas".5 Destaca-se ainda que o "Roteiro"5 respondia às necessidades de informação dos gestores estaduais. Como desvantagens apontam-se a inadequação do material para diferentes níveis de complexidade, onde existem vários profissionais envolvidos na assistência à criança; a atribuição de peso igual para todas as variáveis consideradas, além de questões referentes à estrutura e processo, abordadas indistintamente.

O questionário considerava a ação "implantada" quando todas as questões eram respondidas afirmativamente, "parcialmente implantada", se houvesse concordância com 40 a $90 \%$ das respostas preconizadas e "não implantada", se tivesse menos de $40 \%$ de concordância.

Com a adoção destes pontos de corte bastava uma questão ser discordante para se atribuir o grau de parcialmente implantada. Este critério tornou o instrumento muito rígido na consideração da ação implantada. Por exemplo, uma das perguntas que a maioria dos municípios de médio e pequeno porte deixou de responder positivamente para a ação de Crescimento e Desenvolvimento (CD), foi aquela sobre a existência de equipe multiprofissional envolvida no atendimento à criança .

Para o trabalho de campo foram capacitados os 
coordenadores do programa de cada Diretoria Regional de Saúde (DIRES) com abordagem da importância da avaliação e seus objetivos, assim como o treinamento específico para a aplicação do instrumento e consolidação das respostas.

Nos municípios, a coleta se processou através dos coordenadores de saúde materno-infantil local, enfermeiros em sua grande maioria, previamente treinados pelos coordenadores regionais, que atuaram também como supervisores de campo. Os questionários preenchidos foram enviados para as diretorias regionais e, após consolidados, foram remetidos à coordenação da pesquisa para o processamento e análise dos dados, realizados manualmente.

\section{Resultados}

Houve poucas perdas de informações localizadas em algumas ações. Das 181 unidades selecionadas (6 do município do Recife e 175 dos demais) apenas as regionais I, V e X apresentaram essa falha.

Na I DIRES, duas unidades não responderam ao formulário específico para a ação de Controle das Infecções Respiratórias Agudas (IRA). Na V DIRES, uma unidade não respondeu às questões referentes ao Crescimento e Desenvolvimento, Controle das Doenças Diarréicas e Controle das Infecções Respiratórias Agudas. E uma unidade não respondeu ao instrumento sobre Imunização na X DIRES.

Analisando-se a situação de implantação do PAISC nas unidades de referência do estado (Figura 1) observou-se que os melhores percentuais para ação implantada foram para o Incentivo ao Aleitamento Materno (44,6\%) e Imunização (44,3\%), seguidas do Controle das Doenças Diarréicas $(22,7 \%)$. Os piores resultados foram encontrados para o Controle das Infecções Respiratórias Agudas e Crescimento e Desenvolvimento, $13,8 \%$ e $10,8 \%$ respectivamente com a ação implantada. Em $31 \%$ e $27,8 \%$ das unidades estas mesmas ações foram consideradas não implantadas.

\section{Figura 1}

Distribuição do grau de implantação das ações do PAISC nas unidades de referência do estado de Pernambuco, 1995.

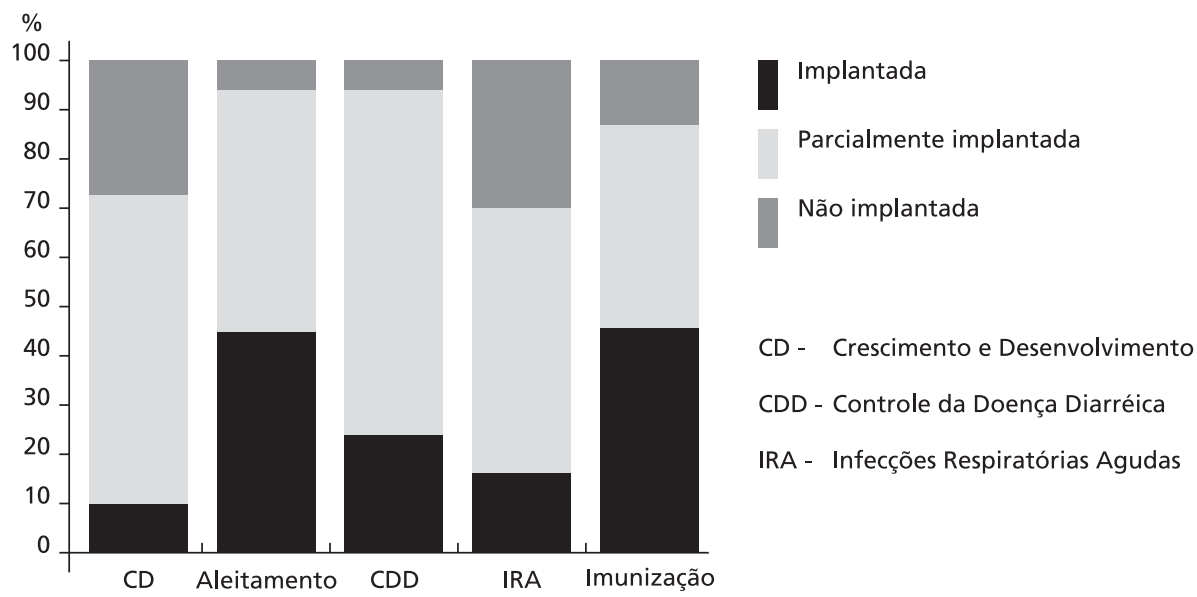

Fonte: Secretaria Estadual de Saúde, 1995 
A Tabela 1 apresenta o número de municípios existentes por Diretoria Regional de Saúde e o número e percentual de unidades de referência com a ação implantada. O primeiro aspecto que chama atenção é a inexistência de unidades na I DIRES, exceto Recife, com as ações de Acompanhamento do Crescimento e Desenvolvimento e Controle das Infecções Respiratórias Agudas implantadas. Além desta, na IX DIRES também não há unidade com a ação de controle das IRA implantada.

A proporção de unidades de referência com o programa implantado variou de acordo com a ação programática. Assim, para o Acompanhamento do Crescimento e Desenvolvimento, os valores oscilaram de $5,9 \%$ na X DIRES a 33,3\% na VIII. Para o Controle das Doenças Diarréicas, de 5,5\% a 50\% na
I e na VIII, respectivamente, e para o Controle das IRA, 5,9\% na $\mathrm{X}$ a $35 \%$ na V. Os melhores resultados foram obtidos para as ações de Imunização e Incentivo ao Aleitamento Materno, a primeira variando de $31,2 \%$ a $63,6 \%$ na X e VII DIRES, respectivamente, e a segunda, de $17,6 \%$ na $X$ e $65,0 \%$ na III DIRES.

Para Recife verificou-se que todas as ações estão implantadas ou parcialmente implantadas. No entanto, nenhuma das seis unidades avaliadas cumpriu integralmente as normas preconizadas pelo Ministério da Saúde (Tabela 2). A melhor situação encontrada foi para a ação de Incentivo ao Aleitamento Materno, implantada em $66,7 \%$ das unidades. Para o Crescimento e Desenvolvimento, três unidades $(50 \%)$, e apenas uma $(16,7 \%)$, com a ação de Controle das IRA implantadas.

Tabela 1

Distribuição de freqüências das Unidades de Saúde das Diretorias Regionais de Saúde segundo ações de saúde da criança implantadas Pernambuco, 1995

\begin{tabular}{|c|c|c|c|c|c|c|c|c|c|c|c|}
\hline \multirow{2}{*}{ DIRES } & \multirow{2}{*}{$\begin{array}{c}\mathbf{N}^{\circ} \text { de } \\
\text { unidades }\end{array}$} & \multicolumn{2}{|c|}{ CD } & \multicolumn{2}{|c|}{ CDD } & \multicolumn{2}{|c|}{ IRA } & \multicolumn{2}{|c|}{ Imunização } & \multicolumn{2}{|c|}{ Aleitamento } \\
\hline & & $n$ & $\%$ & $\mathrm{n}$ & $\%$ & $n$ & $\%$ & $\mathrm{n}$ & $\%$ & $n$ & $\%$ \\
\hline 1 & $17 *$ & - & - & 1 & 5,5 & - & - & 9 & 50,0 & 7 & 38,9 \\
\hline II & 30 & 4 & 13,3 & 6 & 20,0 & 4 & 13,3 & 10 & 33,3 & 11 & 36,7 \\
\hline III & 20 & 2 & 10,0 & 4 & 20,0 & 5 & 25,0 & 12 & 60,0 & 13 & 65,0 \\
\hline IV & 32 & 4 & 12,5 & 4 & 12,5 & 2 & 6,3 & 13 & 40,6 & 17 & 53,1 \\
\hline V & 21 & 3 & 14,3 & 7 & 33,3 & 7 & 33,3 & 13 & 61,9 & 10 & 47,6 \\
\hline VI & 12 & 1 & 8,3 & 4 & 33,3 & 1 & 8,3 & 6 & 50,0 & 7 & 58,3 \\
\hline VII & 11 & 1 & 9,1 & 1 & 9,1 & 3 & 27,3 & 7 & 63,6 & 6 & 54,5 \\
\hline VIII & 6 & 2 & 33,3 & 3 & 50,0 & 1 & 16,7 & 3 & 50,0 & 3 & 50,0 \\
\hline IX & 10 & 1 & 10,0 & 4 & 40,0 & - & - & 5 & 50,0 & 2 & 20,0 \\
\hline $\mathrm{x}$ & 16 & 1 & 5,9 & 6 & 35,3 & 1 & 5,9 & 5 & 31,2 & 3 & 17,6 \\
\hline
\end{tabular}

DIRES = Diretoria Regional de Saúde; $C D=$ Crescimento e Desenvolvimento; $C D D=$ Controle de doenças dearréicas; IRA = Infecções respiratórias agudas.

* Excluídas as unidades do município do Recife, inserido na I DIRES, que foi analisado separadamente conforme se vê na Tabela 2 Fonte: Secretaria Estadual de Saúde, 1995 
Distribuição do grau de implantação das ações de saúde da criança nas seis unidades de referência dos distritos sanitários do Recife, 1995.

\begin{tabular}{llllll}
\hline \multirow{2}{*}{ Ações } & \multicolumn{2}{c}{ Implantada } & \multicolumn{2}{c}{$\begin{array}{c}\text { Parcialmente } \\
\text { implantada }\end{array}$} & Não implantada \\
\cline { 2 - 6 } & $\mathbf{n}$ & $\%$ & $\mathbf{n}$ & $\%$ & $\mathbf{n}$ \\
\hline Crescimento e Desenvolvimento & 3 & 50,0 & 3 & 50,0 & - \\
Incentivo ao Aleitamento Materno & 4 & 66,7 & 2 & 33,3 & - \\
Controle das Doenças Diarréicas & 1 & 16,7 & 5 & 83,3 & - \\
Controle das IRA & 1 & 16,7 & 5 & 83,3 & - \\
Imunização & 3 & 50,0 & 3 & 50,0 & - \\
\hline
\end{tabular}

Fonte: Secretaria Estadual de Saúde, 1995

\section{Discussão}

Este estudo pretendeu conhecer a realidade das ações dos programas de saúde ao mesmo tempo em que se planejava e se interferia na realidade, confirmandose a importância da avaliação como instrumento norteador para o planejamento e reorientação das políticas públicas. 4,7

Foram trabalhados dois dos quatro objetivos de uma avaliação citados por Contandriopoulos et al.4: o primeiro objetivo (estratégico) procuraria contribuir com o planejamento e reestruturar a intervenção voltada para redução da mortalidade infantil. O segundo, formativo, forneceria informações para subsidiar a melhoria da intervenção em curso. Os outros dois, somativo e fundamental, não foram objetos desse estudo.

Com relação ao método, a avaliação tem privilegiado o epidemiológico para diagnosticar, monitorar e observar os resultados de programas e projetos de saúde. No entanto, a maioria dos estudos avalia os efeitos (resultados) desconsiderando a análise de implantação.

A Secretaria de Saúde de Pernambuco, ${ }^{8}$ estudando a mortalidade infantil no Estado, por métodos indiretos, identificou uma taxa de 54,28 óbitos por 1.000 nascidos vivos em 1996. Considerando que o setor saúde deveria ter capacidade de intervir sobre o processo saúde-doença, mesmo em condições de vida adversa, como é o caso dos municípios do estado de Pernambuco, parece que os valores encontrados são compatíveis com o grau de implantação do programa da criança.

No entanto há de se considerar que os resultados encontrados na presente pesquisa se limitam às unidades de referência de Pernambuco, não se podendo fazer extrapolação para o conjunto das unidades dos municípios nem para outras localidades. Porém, o conjunto de normas e padronizações usadas como referencial comparativo pode ser amplamente utilizado em função de sua validade previamente testada.

A seguir será descrita cada ação do programa separadamente.

A ação de Crescimento e Desenvolvimento deveria ser o eixo em torno da qual se desenvolveriam as demais, porém foi onde se observou o pior grau de implantação.

A II Pesquisa Estadual de Saúde e Nutrição, Pernambuco (II PESN) demonstrou que aproximadamente $90 \%$ das mães entrevistadas informavam possuir o "cartão da criança", porém, em menos de $20 \%$ deles havia o registro de peso e apenas em $1 \%$, os marcos do desenvolvimento. ${ }^{9}$ O que se observou na prática é o uso do "cartão da criança" quase que exclusivamente como cartão de vacina, encontrado em $86,3 \%$ dos entrevistados na II PESN. 9 Estas informações parecem compatíveis com os valores identificados neste estudo para o grau de implantação.

Continuando a análise, observou-se que, de todas as ações do PAISC, as que estavam com melhor percentual de implantação eram as de Aleitamento Materno (44,6\%) e Imunização (44,3\%). A II PESN revelou que $10 \%$ das crianças menores de 4 meses estavam em aleitamento materno exclusivo. ${ }^{9}$ Embora se reconheça a multiplicidade dos fatores envolvidos com o aleitamento materno (socioeconômicas e culturais), espera-se que um processo articulado de cuidados primários envolvendo os Agentes Comunitários de Saúde, as unidades básicas, os Hospitais 
"Amigo da Criança" e a mídia, possa ser decisivo na melhoria dos índices encontrados.

Em relação à imunização, o percentual de implantação encontrado (44,3\%) foi baixo. Contraditoriamente, as coberturas vacinais alcançadas nas campanhas e rotinas estão dentro dos padrões aceitáveis pelo Programa Nacional de Imunização (PNI). A existência de alguns casos decorre provavelmente da existência dos bolsões de baixa cobertura, o que levou a Secretaria de Saúde do Estado a adotá-los como evento sentinela.

Outro fato sugestivo de uma implantação melhor do que a encontrada é que a mortalidade por doenças imunopreveníveis em Pernambuco tem decrescido de forma considerável ao longo da década de 80 , atingindo baixas proporções nos anos 90 , porém ainda persiste uma morbidade residual. 10

Estas disparidades talvez sejam explicadas pela pouca validade de conteúdo considerando o número insuficiente de questões para avaliar a ação e talvez também pelo rigor estabelecido pelo método, como já referido anteriormente. Reforçando a afirmação, uma pesquisa realizada em dois municípios do interior do Rio Grande do Norte apontou a ação de Imunização como ponto forte do programa maternoinfantil.11

Quanto ao Controle das Doenças Diarréicas a ação está implantada em 22,7\% das unidades estudadas. Este baixo percentual, provavelmente, foi um dos determinantes da situação encontrada na II PESN, no que concerne ao manejo da diarréia nos menores de 5 anos. Esta verificou que 35,6\% das crianças que apresentaram diarréia nas duas semanas anteriores à entrevista usaram medicação e 22,8\% suspenderam a alimentação durante o episódio diarréico. ${ }^{9}$ No entanto, o uso dos Sais de Reidratação Oral (SRO) está universalizado, ${ }^{9}$ possivelmente devido às campanhas de divulgação veiculadas através da mídia, aos agentes comunitários de saúde e ao trabalho desenvolvido nas unidades de saúde.

Com relação ao Controle das Infecções Respiratórias Agudas, foi detectado o mais alto percentual de unidades $(31 \%)$ com a ação não implantada. Uma das questões abordadas pelo instrumento foi a utilização de normas e cartazes padronizados para o manejo dos casos de IRA, que foi respondida, pela maioria, negativamente. Dois outros estudos realizados em 1996 e 1997, analisando o programa de controle das IRA, no Recife, mostraram que apenas $30 \%$ e $16 \%$ das unidades dispõem destas normas nos serviços. 12,13 Com relação aos casos de pneumonia, menos da metade foram tratados de acordo com a norma. Considerando-se o tratamento antibiótico das infecções respiratórias agudas, $49 \%$ das prescrições poderiam ter sido evitadas se os critérios do programa tivessem sido utilizados. 12,13

Hartz et al. ${ }^{14}$ avaliaram o grau de implantação do programa materno-infantil em dois municípios do Rio Grande do Norte, utilizando a doença diarréica e as infecções respiratórias agudas como condições traçadoras da qualidade dos cuidados médicos. 10,14 Apesar do estudo se diferenciar metodologicamente deste, a análise da implantação dessas ações mostrou-se insatisfatória em ambos os municípios.

Os diferentes graus de implantação encontrados neste estudo poderiam estar relacionados aos investimentos em recursos humanos e equipamentos, a mudança dos gestores do nível municipal, supervisões sistemáticas e decisão política. Aliada a isso ainda tem-se a decisão individual dos profissionais na aplicação das normas preconizadas. As práticas programáticas em saúde pressupõem a padronização da investigação, critérios diagnósticos e conduta. A simplificação que daí advém permite, entre outras coisas, a homogeneidade dos esquemas terapêuticos tornando mais factível a sua utilização. Objetiva a obtenção de resultados semelhantes por diferentes profissionais e, face a menor variabilidade dos procedimentos, facilita a avaliação do impacto dos mesmos. É exatamente a possibilidade de uma avaliação externa e a restrição aos atos técnicos do médico que pode acarretar uma resistência ao programa por esse profissional.

Em um processo de avaliação deve-se ter em mente que múltiplos fatores estão envolvidos na determinação dos resultados. A avaliação normativa enfatiza apenas uma das faces do problema, o que deve ser considerado para que não se faça julgamento precipitado. No entanto, este tipo de avaliação parece etapa obrigatória no monitoramento e condução de programas. 


\section{Referências}

1. Pernambuco. Secretaria de Saúde. Avaliação das ações de atenção integral à saúde da criança. Recife: Secretaria Estadual de Saúde; 1996. (Caderno de Avaliação, Série Projeto Salva-Vidas, 4).

2. Ministério da Saúde. Metas da cúpula mundial em favor da infância - avaliação de meia década: 1990 -1995. Brasília, DF: Ministério da Saúde; 1997.

3. Pernambuco. Secretaria de Saúde, UNICEF. Fundo das Nações Unidas para a Infância. Crianças e adolescentes em Pernambuco. Saúde, educação e trabalho. Recife: Secretaria Estadual de Saúde; 1992. p: 5-54.

4. Contandriopoulos A-P, Champagne F, Denis J, Pineault R A avaliação na área da saúde: conceitos e métodos. In: Hartz ZMA, organizadora. Avaliação em saúde: dos modelos conceituais à prática na análise da implantação de programas. Rio de Janeiro: FIOCRUZ. Fundação Oswaldo Cruz; 1997. p. 29-47.

5. Ministério da Saúde. Secretaria de Assistência à Saúde. Roteiro de avaliação dos programas PAISM/PAISC/PROSAD. Brasília, DF: Ministério da Saúde; 1994.

6. Contandriopoulus A-P, Champagne F, Potvin L, Denis JL, Boyle P. Saber preparar uma pesquisa - definição, estrutura e financiamento. São Paulo: HUCITEC; 1994.

7. Castiel LD. Considerações acerca da utilização da epidemiologia na avaliação dos sistemas de saúde. Cad Saúde Pública 1986; 2: 184-90.

8. Pernambuco. Secretaria de Saúde. AIDPI - Atenção de Integrada às Doenças Prevalentes na Infância - processo de implantação da estratégia em Pernambuco. Recife: Secretaria Estadual de Saúde; 1998. (Caderno de Avaliação. Série Projeto Salva-Vidas, 14)

9. Ministério da Saúde. INAN. Instituto Nacional de Alimentação e Nutrição, IMIP. Instituto Materno Infantil de Pernambuco, Universidade Federal de Pernambuco, Departamento de Nutrição, Pernambuco. Secretaria de Saúde. Pesquisa estadual de saúde e nutrição. Recife: Ministério da Saúde; 1998
10. Pernambuco. Secretaria de Saúde. Doenças infecciosas e suas vacinas: uma breve revisão da história recente: 1980-1997. Recife; Secretaria Estadual de Saúde; 1998. [mimeo]

11. Hartz ZMA, Champagne F, Contandriopoulus A-P, Leal MC. Avaliação do programa materno infantil: análise de implantação em sistemas locais de saúde no Nordeste do Brasil. In: Hartz ZMA, organizadora. Avaliação em saúde: dos modelos conceituais a prática na análise da implantação de programas. Rio de Janeiro: FIOCRUZ., Fundação Oswaldo Cruz; 1997. p. 89-131.

12. Vidal SA. Avaliação do programa de controle das infecções respiratórias agudas nos serviços públicos ambulatoriais da cidade do Recife [dissertação mestrado]. Recife: Instituto Materno Infantil de Pernambuco, IMIP; 1996.

13. Pernambuco. Secretaria de Saúde. Avaliação sobre o manejo das infecções respiratórias agudas em menores de 5 anos no município de Recife/PE. Recife: Secretaria Estadual de Saúde; 1998. (Caderno de Avaliação. Série Projeto Salva-Vidas, 10).

14. Hartz ZMA, Champagne F, Contandriopoulos A-P, Leal MC. Uso de traçadores para avaliação de qualidade da assistência à criança: o controle da doença diarréica e das infecções respiratórias agudas em dois centros de saúde. Revista do IMIP 1995; 9: 35-50. 